\title{
FutureJournal
}

\section{E-Serviços no Governo Eletrônico: Análise Bibliométrica de Artigos Internacionais}

Marcus Vinicius Moreira Zittei

Doutorando em Ciências Contábeis pela Universidade Regional de Blumenau (FURB), Brasil marcuszittei@zittei.com.br

\section{Francisco Carlos Fernandes}

Doutor em Controladoria e Contabilidade pela Universidade de São Paulo (USP), Brasil fernandes.francisco.0208@gmail.com

\section{RESUMO}

Neste estudo, o objetivo foi analisar a produção e o perfil das publicações do tema ferramentas de e-Serviços referente a governo eletrônico na base de dados Scopus, no período de 2001 a 2015. O governo eletrônico compreende ferramentas para relação do governo com a sociedade, cidadãos, outros governos e negócio. $\mathrm{O}$ foco desses artigos compreende e-serviços e atividades de governo eletrônico para os negócios. Foi utilizada pesquisa descritiva, conduzida por meio de levantamento bibliográfico, com abordagem bibliométrica e análise quantitativa, com amostra coletada na base de dados Scopus. Concluiu-se que o número de trabalhos nesse período foi de 299, sendo 45 trabalhos publicados em eventos em 2010 e 16 artigos publicados em revistas em 2013. O ponto central dos artigos com maior citação está relacionado à implantação e à percepção de usuários de ferramentas implantadas por países.

PALAVRAS-CHAVE: Governo Eletrônico. E-Serviços. E-Governo. Bibliometria. 


\section{FutureJournal}

\section{E-Service in E-Government: Bibliometric Analysis International Papers}

\section{ABSTRACT}

This paper aimed to analyze the production and the profile of the publications of E-Services tools theme related to e-government in Scopus database from 2001 to 2015 . E-government understand tools for government's relationship to society, citizens, other governments and business. The focus of papers understand E-services, egovernment activities for business. Utilized descriptive research, conducted through bibliographic survey, to bibliometric approach and quantitative analysis, to sample collected in Scopus database. It concluded that the number of papers in this period was 299, with 45 papers published in events in 2010 and 16 papers published in journals in 2013. The focus of the papers with the highest citation related to the implementation and realization of established tools users by countries.

KEY-WORDS: Electronic Government. E-services. E-government. Bibliometric. 


\section{INTRODUÇÃO}

As constantes mudanças relativas às novas tecnologias de informação e de comunicação (TIC) afetam a sociedade de forma geral e direta, assim como os governos em seus processos de tomada de decisão. Em vista dessa realidade, governantes de distintos países estão ou aplicando ou preparandose para utilizar essas ferramentas na construção de governos mais democráticos, para estreitar o relacionamento do setor público com a sociedade civil.

A ideia de e-governo surge para suprir essa necessidade, utilizando as TICs de forma a alcançar o objetivo de democratizar os governos e haver maior transparência e controle social.

Para Diniz, Barbosa, Junqueira \& Prado (2009), o uso das TICs está associado à utilização da internet para a prestação de serviços eletrônicos, podendo essa tecnologia ser utilizada seja pelos governos como estratégia em seus processos internos e na melhoria dos serviços à sociedade, seja pelos cidadãos, seja pelas pessoas jurídicas. Nesse sentido, a utilização da internet e das novas tecnologias de informação pela administração pública como instrumento de reforma e modernização deu início ao termo "governo eletrônico".

Ainda segundo Chen, Chen, Huang \& Ching (2006), o governo eletrônico (e-gov) foi criado para integrar governo, empresas e cidadãos por meio da tecnologia da informação e comunicação.

No entanto, cabe ressaltar que o governo eletrônico não está vinculado somente à utilização das TICs, pois, de acordo com Agune \& Carlos (2005), o governo eletrônico, ao contrário do que o nome pode a princípio sugerir, significa muito mais do que a intensificação do uso da tecnologia da informação pelo poder público, ele deve ser encarado como a transição de uma forma de governar fortemente segmentada, hierarquizada e burocrática, para outra mais horizontal, colaborativa, flexível e inovadora.

Nesta pesquisa, o objetivo é analisar a produção e o perfil das publicações do tema ferramentas de e-serviços referente a governo eletrônico na base de dados Scopus, no período de 2001 a 2015.

Por ser um tema cujos artigos publicados vêm aumentando com o passar dos anos, justifica-se analisar a produção e o perfil intelectual de 
publicações com relação ao e-serviços como ferramenta do governo eletrônico utilizada nas empresas nos períodos de 2001 a 2015, para assim conhecer as aplicações práticas em diversas realidades e os impactos nas organizações tanto públicas quanto privadas. Justifica-se também como contribuição à consolidação teórica do campo de governo eletrônico.

No contexto exposto, a questão é: qual a produção e o perfil intelectual de publicações dos artigos na base Scopus no tema e-serviços como ferramenta do governo eletrônico utilizada nas empresas nos períodos de 2001 a 2015?

O trabalho está dividido em cinco partes. Primeiramente a introdução, em que se apresentam o tema proposto, o objetivo da pesquisa e a problemática. Na segunda, aborda-se a teoria que auxilia o entendimento dos conceitos de governo eletrônico e suas práticas. Na terceira parte, descrevese o método a ser utilizado. No quarto item, são apresentados os resultados da pesquisa e, por fim, vêm as considerações finais.

\section{FUNDAMENTAÇÃO TEÓRICA}

\subsection{GOVERNO ELETRÔNICO}

Segundo Zweers e Planqué (2001, p. 92),

Governo Eletrônico é um conceito emergente que objetiva fornecer ou tornar disponíveis informações, serviços ou produtos, por meio eletrônico, a partir ou por meio de órgãos públicos, a qualquer momento, local e cidadão, de modo a agregar valor a todos os stakeholders envolvidos com a esfera pública.

O governo eletrônico ou e-governo, é uma concepção que assimila o potencial das TICs na transformação da administração pública, com substancial melhoria de sua organização, de seus serviços e do relacionamento com a sociedade. Essa concepção insere-se em uma perspectiva avançada de governança democrática, transparente e aberta à participação do cidadão (Knight, Fernandes \& Cunha, 2007).

Percebe-se que o conceito fica mais amplo e requer maior esforço dos governos do que somente a utilização de tecnologias em suas práticas. A qualidade, a eficiência na prestação de serviço para a sociedade e os 
processos de transparência ganham destaque e o modelo dissemina-se em escala global. Cunha (2005) argumenta que conceitos mais abrangentes de e-gov passaram a ser considerados, envolvendo diversos tópicos: melhoria dos processos de gestão pública, eficiência e efetividade, melhor governança, elaboração e monitoramento das políticas públicas, prestação de serviços e democracia eletrônica, esta última englobando transparência, participação e accountability.

O estudo do governo eletrônico, segundo Helbig, Gil-García e Ferro (2005), pode ser dividido em três tipos de relações:

> G2G (Government to Government), quando se trata de uma relação intra ou intergovernos;

> G2C (Government to Citizen), relações entre governo e cidadão, diz respeito às ações que o governo realiza no sentido de colocar à disposição do cidadão, por meio eletrônico, serviços e informações pertinentes à esfera pública;

> G2B (Government to Business), caracterizado por transações entre governos e fornecedores ou pessoas jurídicas. Também conhecidos como e-serviços (e-service)

Criando canais eletrônicos de informações e disponibilizando serviços à sociedade, o governo eletrônico surge como um importante instrumento de gestão pública que possibilita ainda mais controle e transparência.

Ao exporem os esforços empreendidos no mundo para a implantação do governo eletrônico, Silva e Lima (2007) afirmam que parece haver um entendimento mundial de que este é o caminho para dinamizar a relação entre governo e cidadão e promover a democratização eletrônica.

De acordo com Knight et al. (2007, p. 492), a política do governo eletrônico é orientada pelas seguintes diretrizes:

$>$ oferta de serviços e informações ao cidadão;

> promoção de acesso da população à internet;

$>$ integração entre sistemas de informação, redes, bancos de dados governamentais;

> implementação de uma infraestrutura avançada de comunicações e serviços na administração pública; 
$>$ utilização do poder de compra do governo federal para a redução de custos.

Segundo Pessi (2007), a implementação de iniciativas orientadas para a melhoria do relacionamento entre governo e cidadãos pelo uso das TICs convive permanentemente com a busca do equilíbrio entre pelo menos três fatores, quando o assunto em questão é a disponibilização de serviços públicos por meios eletrônicos. De um lado estão as prioridades estabelecidas pelos governos, em função de suas necessidades de gestão. De outro, as expectativas da sociedade, que espera eficiência, agilidade e obtenção de benefícios. Por fim, resta o desafio do canal de distribuição de serviços e conteúdos eletrônicos dadas as desigualdades de acesso à internet e outros dispositivos necessários ao sucesso da interação.

Desde 1990, nota-se que os governos, em diferentes níveis, iniciaram - lançamento de projetos de governo eletrônico, para disponibilizar informações e serviços eletrônicos aos cidadãos e às empresas (Mello, 2009).

A sociedade tendo acesso às TICs, possibilita-se que qualquer pessoa obtenha informações relacionadas a si e aos outros, o que representa ampliação de canais importantes em países em desenvolvimento, pois concede-se a seus usuários acesso quase ilimitado à base de dados de seus interesses (Mello, 2009).

Com relação às TICs, sua adoção possibilita ir além da informação passiva, porque envolve a participação ativa dos cidadãos no processo de tomada de decisão (Unesco, 2005).

Os avanços nas tecnologias de informação e comunicação têm propiciado implementação de novas aplicações e da prestação de alta qualidade nos serviços através das redes globais; o objetivo é utilizar essa área da informação à sociedade para melhoria na qualidade de vida de todos os cidadãos, ampliando o conhecimento, os ganhos sociais e mantendo as entidades públicas atualizadas no cenário global eletrônico (Santhanamery \& Ramayah, 2012).

Mello (2009, p. 39) comenta que "com o advento das TICs, a governança eletrônica aparece como uma tendência emergente para reinventar o funcionamento do governo, especialmente na oferta de serviços públicos e participação cidadã na gestão, de maneira on-line". 
Apesar de fortemente ligado ao uso da tecnologia de informação no setor público, o termo governo eletrônico não se limita a esse elemento, na verdade vincula-se a uma modernização ampla no âmbito da administração pública, na melhoria da eficiência de seus processos operacionais e administrativos, que representam a transição de uma forma de governar segmentada, hierarquizada e burocrática, para um Estado mais horizontal, colaborativo, flexível e inovador, mais coerente com a chegada da sociedade do conhecimento (Agune \& Carlos, 2005).

\subsubsection{Governo eletrônico no Brasil e no mundo}

O marco histórico mundial para o alcance do governo eletrônico foi em agosto de 1993, quando houve o lançamento do Mosaica, primeiro navegador que permitiu uma navegação fácil pela web. Contudo, o movimento formalizou-se em 15 janeiro de 1999, com a abertura do 10 Fórum Global sobre Reinvenção do Governo, em Washington, com a presença de representantes de 45 países, entre eles o Brasil (Chahin, Cunha, Knight \& Pinto, 2004).

Segundo Geron, Finatelli, Faria e Romeiro (2011), o e-governo foi introduzido a partir da possibilidade de utilização das TICs para apoiar, modernizar e melhorar os serviços públicos.

De acordo com Menezes (2012), no Brasil, o governo eletrônico foi instituído por decreto pelo então presidente Fernando Henrique Cardoso no ano de 2000, quando foi criado um Grupo de Trabalho Interministerial para examinar e propor políticas, diretrizes e normas relacionadas às novas formas de interação eletrônica.

Entre os países que criaram legislação sobre o assunto, estão Argentina, Chile, México, Costa Rica, Colômbia e Austrália, além dos países da União Europeia, em função da adoção da Diretiva 115/2001.

Em 2001, o Chile lançou sua estratégia de governo eletrônico. Os esforços em torno da implantação desse novo sistema no país têm sido reconhecidos não só nacional, mas também internacionalmente. Há uma série de estudos internacionais que colocam o Chile nos primeiros lugares em 
relação ao desenvolvimento do e-governo em todo o mundo (Sepúlveda, Vásquez \& Gutiérres, 2006).

O Chile foi um dos primeiros países na América Latina a implantar em seu governo a validação dos documentos eletrônicos por meio de um certificado digital. Em março de 2002, promulgou-se a Lei no. 19.799 desse país, que trata sobre documentos eletrônicos, assinaturas eletrônicas e certificação de serviços das empresas.

Assim como o Chile, o México também foi um dos pioneiros em desenvolver a certificação digital para documentos eletrônicos, em janeiro de 2002, por meio de um decreto. O objetivo era regulamentar a certificação por meio de identificação eletrônica, para ser utilizada em transações eletrônicas. Por meio desse decreto, o governo autoriza as empresas devidamente regulamentadas a emitir a certificação digital.

Para Geron et al. (2011), o governo brasileiro também está empenhado em melhorar seus serviços públicos por meio das TICs através de sistemas de relacionamento com contribuintes, uma vez que se inicia uma nova era na informática que fará parte também do quotidiano dos empresários, advogados, contabilistas e demais profissionais.

Aproveitando esse momento de evolução, em termos de gestão, o governo brasileiro utilizou a experiência de governos eletrônicos de outros países como modelo, tais como Espanha, Chile e México, e também entrou na era digital, conforme mencionado por Britto (2008), com rotinas de transmissão de dados com assinatura digital, manipulação de arquivos eletrônicos, etc. Diante disso, surgiu o Sistema Público de Escrituração Digital (SPED), com a finalidade de aproximar o fisco de seus contribuintes (Brasil, 2009).

Esse sistema foi instituído pelo governo brasileiro no ano de 2007 e faz parte do Programa de Aceleração do Crescimento (PAC). De acordo com Matos, Costa, Pereira e Locks (2008), o SPED nasceu com o objetivo de promover a integração dos sistemas de fiscalização e arrecadação, uniformizando as obrigações acessórias para os contribuintes e simultaneamente tornando mais rápida a identificação de ilícitos tributários. 


\subsubsection{E-administração, e-serviços e e-democracia}

Com o objetivo de reconhecer e visualizar sob quais formas são demonstradas as ações de governança eletrônica, é importante segregá-la em três grandes ramos: e-administração, compreendida como o uso dos meios eletrônicos pela administração pública; e-serviços, que representa a prestação de serviços ao cidadão e às empresas; e e-democracia, o uso dos meios eletrônicos no suporte à prática democrática.

Abordando o primeiro tema, e-administração, trata-se do suporte digital à elaboração e implementação de políticas públicas. A tecnologia da informação auxilia não só a implementação da política pública, a tomada de decisão, mas também o monitoramento da implementação, o controle da política e a avaliação dos resultados, em termos da aplicação dos recursos, mas, principalmente, na efetividade da política implementada. Também, a tecnologia pode fazer o trabalho de suporte de comunicação, de workgroup, e viabilizar a eficiência interna de processos - como processos de compra, viagens de servidores, recursos humanos, controle de receitas e despesas, acompanhamento do planejamento governamental, e outros. Ainda em eadministração, pode-se incluir a integração de políticas públicas entre as várias esferas de governo. Ao falar agora de e-administração e transparência, no Brasil, um exemplo óbvio de resultados alcançados foi a Lei de Responsabilidade Fiscal (LRF), quando exigiu que todos os demonstrativos governamentais fossem publicados na internet (Cunha, 2005, p. 2).

Sobre a ramificação da governança eletrônica denominada e-serviços, Ruelas e Arâmburo (2006) comentam que o crescimento e a melhoria dos serviços estão ligados diretamente, em grande parte, aos múltiplos benefícios que retornam ao governo, aos cidadãos e às empresas, especialmente tornando mais ágeis e eficazes os procedimentos, afastando a burocracia e reduzindo os custos nas transações, transformando os recursos de forma produtiva por meio de respostas mais rápidas, ampliação da cobertura e qualidade nos serviços. O resultado é um relacionamento mais assertivo entre governo, cidadãos e empresas.

Como exemplificação do conceito de e-serviços, Cunha (2005) cita a figura das centrais de atendimento. Com o suporte de recursos tecnológicos e canais de comunicação, essas centrais são capazes de oferecer - em nível municipal, estadual ou federal - facilidades como o atendimento com qualidade ao cidadão e às empresas. Assim, qualquer um poderá ter sua respectiva solicitação atendida, limitados apenas os casos em que há necessidade de especialidades, como os serviços de saúde. Um exemplo 
dessas centrais de atendimento chama-se Poupatempo, no estado de São Paulo.

Sobre o terceiro ramo da governança eletrônica, denominado edemocracia ou democracia eletrônica, com o advento das novas tecnologias de informação e comunicações, foi possível obter ampliação das discussões e efetividade da participação da sociedade nos processos de políticas públicas (Mezzaroba \& Galindo, 2010).

Rezende, Frey e Betini (2006) ressaltam que o uso de estratégias democráticas de gestão e de tecnologia da informação e comunicação pelos mais diversos atores democráticos (governo, representantes oficiais eleitos, mídia, organizações políticas, cidadãos) dentro dos processos políticos e de governança de comunidades caracteriza a democracia eletrônica.

\section{METODOLOGIA}

A pesquisa caracteriza-se como descritiva, conduzida por meio de levantamento bibliográfico, com abordagem bibliométrica e análise quantitativa. Classifica-se, pois, como bibliográfica, porque tem como objetivo analisar a produção e o perfil das publicações do tema ferramentas de e-serviços referente a governo eletrônico na base de dados Scopus importante base de dados internacional (Vanz \& Stumpf, 2010), com indexação das principais revistas de governo eletrônico -, no período de 2001 a 2015. O período escolhido remete às primeiras publicações com a temática e-serviços até janeiro de 2015 , data da pesquisa final.

Segundo Vanti (2002, p. 153), uma pesquisa bibliométrica

"é um conjunto de métodos de pesquisa que utiliza análise quantitativa, estatística e de visualização de dados, objetivando mapear a estrutura do conhecimento de um campo científico e servir de ferramenta para análise do comportamento do pesquisador na construção do conhecimento".

A amostra deste estudo refere-se aos 299 artigos apresentados na área temática "E-Serviços" disponível na base Scopus no período de 2001 a 2015. Utilizou-se como critério de busca o período temporal, buscou-se no título dos artigos o termo e-government, limitado pelas palavras-chave e-government services, e-services e government services. 
No processo de coleta de dados, após seleção dos artigos, foi realizada, por meio de planilha eletrônica do Microsoft Excel, a tabulação dos dados, na qual destacaram-se os seguintes itens: quantidade de artigos publicados no período de 2001 a 2015, identificação dos autores, análise dos autores, análise das citações, análise das publicações. Utilizaram-se as funcionalidades disponibilizadas pela base de dados Scopus.

\section{APRESENTAÇÃO E ANÁLISE DOS RESULTADOS}

Primeiramente, apresenta-se a evolução dos artigos no período de 2001 a 2015, separados por publicações em revistas e eventos. Em seguida apresenta-se a distribuição por país, área de estudo, total de citações por autores, artigos mais citados, total de publicações por revista e por eventos.

Na Tabela 1, apresenta-se a evolução da quantidade de artigos por tipo de publicação (revistas ou eventos) no período de 2001 a 2015.

Tabela 1: Evolução da quantidade de artigos publicados

\begin{tabular}{c|c|c|c|c}
\hline Ano & Quantidade Total & Revistas & Eventos & \% \\
\hline 2001 & 1 & 0 & 1 & $0,33 \%$ \\
\hline 2002 & 0 & 0 & 0 & $0,00 \%$ \\
\hline 2003 & 2 & 0 & 2 & $0,67 \%$ \\
\hline 2004 & 4 & 1 & 3 & $1,34 \%$ \\
\hline 2005 & 14 & 4 & 10 & $4,68 \%$ \\
\hline 2006 & 9 & 3 & 6 & $3,01 \%$ \\
\hline 2007 & 21 & 4 & 17 & $7,02 \%$ \\
\hline 2008 & 34 & 9 & 25 & $11,37 \%$ \\
\hline 2009 & 52 & 10 & 42 & $17,39 \%$ \\
\hline 2010 & 57 & 12 & 45 & $19,06 \%$ \\
\hline 2011 & 45 & 10 & 35 & $15,05 \%$ \\
\hline 2012 & 28 & 11 & 17 & $9,36 \%$ \\
\hline 2013 & 21 & 16 & 5 & $7,02 \%$ \\
\hline 2014 & 10 & 5 & 5 & $3,34 \%$ \\
\hline 2015 & 1 & 1 & 0 & $0,33 \%$ \\
\hline Total Geral & $\mathbf{2 9 9}$ & $\mathbf{8 6}$ & $\mathbf{2 1 3}$ & \\
\hline
\end{tabular}

Fonte: Dados da pesquisa

Pode-se observar na Tabela 1, o maior número de publicações em eventos, por ser um tema cujas aplicações iniciaram-se na década de 1990 (Mello, 2009). Do total de 213 publicações em eventos e 86 em periódicos, 
no ano de 2010, apresentou-se o maior número de publicações em eventos (45); no ano 2013, apresentaram-se 16 artigos em revistas.

$\mathrm{Na}$ Tabela 2, apresenta-se o número de publicações por país de origem dos autores. Reino Unido, Estados Unidos da América e China apresentam os maiores índices, 39, 34 e 30, respectivamente; o Brasil apresentou três trabalhos.

Tabela 2: Número de publicações por país

\begin{tabular}{l|c|c|c|c}
\hline \multicolumn{1}{c|}{ Ano } & Quantidade Total & Revistas & Eventos & \% \\
\hline United Kingdom & 39 & 14 & 25 & $11,47 \%$ \\
\hline United States & 34 & 13 & 21 & $10,00 \%$ \\
\hline Greece & 19 & 13 & 6 & $5,59 \%$ \\
\hline Taiwan & 11 & 6 & 5 & $3,24 \%$ \\
\hline Spain & 9 & 6 & 3 & $2,65 \%$ \\
\hline Sweden & 12 & 5 & 7 & $3,53 \%$ \\
\hline Netherlands & 8 & 5 & 3 & $2,35 \%$ \\
\hline Australia & 15 & 4 & 11 & $4,41 \%$ \\
\hline Canada & 4 & 4 & 0 & $1,18 \%$ \\
\hline Germany & 9 & 3 & 6 & $2,65 \%$ \\
\hline Malaysia & 10 & 3 & 7 & $2,94 \%$ \\
\hline Italy & 11 & 3 & 8 & $3,24 \%$ \\
\hline France & 7 & 2 & 5 & $2,06 \%$ \\
\hline Iran & 10 & 2 & 8 & $2,94 \%$ \\
\hline Ireland & 6 & 2 & 4 & $1,76 \%$ \\
\hline Lithuania & 7 & 2 & 5 & $2,06 \%$ \\
\hline Romania & 5 & 1 & 3 & $1,47 \%$ \\
\hline Brazil & 3 & 1 & 2 & $0,88 \%$ \\
\hline China & 31 & $\mathbf{1 0 6}$ & $\mathbf{2 3 4}$ & $9,12 \%$ \\
\hline Outros & 90 & & 75 & $26,47 \%$ \\
\hline Total Geral & $\mathbf{3 4 0}$ & & \\
\hline
\end{tabular}

Fonte: Dados da Pesquisa

$\mathrm{Na}$ Tabela, 3 apresentam-se as áreas de estudo de vinculação dos eventos e revista. Ciências da Computação apresentou o maior índice $(61,76 \%)$, seguida por Negócios, Gestão e Contabilidade (43,82\%). 
Tabela 3: Áreas de estudo

\begin{tabular}{l|c|c|c|c}
\hline \multicolumn{1}{c|}{ Ano } & Quantidade Total & Revistas & Eventos & $\mathbf{\%}$ \\
\hline Ciências Sociais & 106 & 62 & 44 & $31,18 \%$ \\
\hline Ciências da Computação & 210 & 61 & 149 & $61,76 \%$ \\
\hline Negócios, Gestão e Contabilidade & 149 & 19 & 130 & $43,82 \%$ \\
\hline Ciências da Decisão & 88 & 16 & 72 & $25,88 \%$ \\
\hline Engenharia & 21 & 7 & 14 & $6,18 \%$ \\
\hline Economia, Econometria e Finanças & 8 & 4 & 4 & $2,35 \%$ \\
\hline Psicologia & 4 & 4 & 0 & $1,18 \%$ \\
\hline Artes e Humanidades & 3 & 3 & 0 & $0,88 \%$ \\
\hline Matemática & 8 & 0 & 8 & $2,35 \%$ \\
\hline Ciências Ambientais & 1 & 0 & 1 & $0,29 \%$ \\
\hline Total Geral & $\mathbf{5 9 8}$ & $\mathbf{1 7 6}$ & $\mathbf{4 2 2}$ & \\
\hline
\end{tabular}

Fonte: Dados da pesquisa

Na Tabela 4, apresenta-se o total de citações por autores. Todos os trabalhos foram citados 1044 vezes, sendo o de Hung, Chang e Yu o mais citado (158 vezes), seguido pelo de Horst, Kuttschreuter e Guutteling (129 vezes).

Tabela 4: Autores $x$ total de citações

\begin{tabular}{l|c}
\hline \multicolumn{1}{c|}{ Autores } & Total de citações \\
\hline Hung, S.-Y., Chang, C.-M., \& Yu, T.-J. & 158 \\
\hline Horst, M., Kuttschreuter, M., \& Gutteling, J. M. & 129 \\
\hline Tung, L. L., \& Rieck, O. & 105 \\
\hline Lean, O.K., Zailani, S., Ramayah, T., \& Fernando, Y. & 86 \\
\hline Weerakkody, V., \& Dhillon, G. & 61 \\
\hline Steyaert, J.C. & 46 \\
\hline Ghapanchi, A., Albadvi, A., \& Zarei, B. & 30 \\
\hline Kamal, M. M., Weerakkody, V., \& Jones, S. & 23 \\
\hline van Velsen, L., van der Geest, T., ter Hedde, M., \& Derks, W. & 22 \\
\hline Panopoulou, E., Tambouris, E., \& Tarabanis, K. & 21 \\
\hline Lee, J., \& Rao, H. R. & 18 \\
\hline Apostolou, D., Mentzas, G., Stojanovic, L., Thoenssen, B., \& Pariente Lobo, T. & 17 \\
\hline Luk, S. C. Y. & 17 \\
\hline Xiong, J. A. & 15 \\
\hline Sipior, J. C., Ward, B. T., \& Connolly, R. & 14 \\
\hline Seng, W. M., Jackson, S., \& Philip, G. & 14 \\
\hline Hung, S.-Y., Chang, C.-M., \& Kuo, S.-R. & 13 \\
\hline Choudrie, J., Grey, S., \& Tsitsianis, N. & 12 \\
\hline Teerling, M. L., \& Pieterson, W. & 11 \\
\hline Outros & 232 \\
\hline Total Geral & $\mathbf{1 0 4 4}$ \\
\hline
\end{tabular}

Fonte: Dados da pesquisa 
Na Tabela 5, apresentam-se os dez trabalhos publicados mais citados no período entre 2004 e 2009. As publicações Government Information Quarterly e International Journal of Information Management aparecem com dois artigos cada, entre os mais citados.

Tabela 5: Artigos mais citados

\begin{tabular}{|c|c|c|c|c|}
\hline Autores & $\begin{array}{l}\text { Total de } \\
\text { citações }\end{array}$ & Título & Publicação & Ano \\
\hline $\begin{array}{l}\text { Hung, S.-Y., Chang, } \\
\text { C.-M., \& Yu, T.-J. }\end{array}$ & 158 & $\begin{array}{l}\text { Determinants of user acceptance of } \\
\text { the e-government services: The case } \\
\text { of online tax filing and payment } \\
\text { system }\end{array}$ & $\begin{array}{l}\text { Government } \\
\text { Information } \\
\text { Quarterly }\end{array}$ & 2006 \\
\hline $\begin{array}{l}\text { Horst, M., } \\
\text { Kuttschreuter, M., \& } \\
\text { Gutteling, J.M. }\end{array}$ & 129 & $\begin{array}{l}\text { Perceived usefulness, personal } \\
\text { experiences, risk perception and trust } \\
\text { as determinants of adoption of e- } \\
\text { government services in The } \\
\text { Netherlands }\end{array}$ & $\begin{array}{l}\text { Computers in } \\
\text { Human Behavior }\end{array}$ & 2007 \\
\hline Tung, L. L., \& Rieck, O. & 105 & $\begin{array}{l}\text { Adoption of electronic government } \\
\text { services among business } \\
\text { organizations in Singapore }\end{array}$ & $\begin{array}{l}\text { Journal of } \\
\text { Strategic } \\
\text { Information } \\
\text { Systems }\end{array}$ & 2005 \\
\hline $\begin{array}{l}\text { Lean, O. K., Zailani, } \\
\text { S., Ramayah, T., \& } \\
\text { Fernando, Y. }\end{array}$ & 86 & $\begin{array}{l}\text { Factors influencing intention to use e- } \\
\text { government services among citizens } \\
\text { in Malaysia }\end{array}$ & $\begin{array}{l}\text { International } \\
\text { Journal of } \\
\text { Information } \\
\text { Management }\end{array}$ & 2009 \\
\hline $\begin{array}{l}\text { Weerakkody, V., \& } \\
\text { Dhillon, G. }\end{array}$ & 61 & $\begin{array}{l}\text { Moving from e-government T- } \\
\text { government: A study of process } \\
\text { reengineering challenges in a UK local } \\
\text { authority context }\end{array}$ & $\begin{array}{l}\text { International } \\
\text { Journal of } \\
\text { Electronic } \\
\text { Government } \\
\text { Research } \\
\end{array}$ & 2008 \\
\hline Steyaert, J. C. & 46 & $\begin{array}{l}\text { Measuring the performance of } \\
\text { electronic government services }\end{array}$ & $\begin{array}{l}\text { Information and } \\
\text { Management }\end{array}$ & 2004 \\
\hline $\begin{array}{l}\text { Ghapanchi, A., } \\
\text { Albadvi, A., \& Zarei, B. }\end{array}$ & 30 & $\begin{array}{l}\text { A framework for e-government } \\
\text { planning and implementation }\end{array}$ & $\begin{array}{l}\text { Electronic } \\
\text { Government }\end{array}$ & 2008 \\
\hline $\begin{array}{l}\text { Kamal, M. M., } \\
\text { Weerakkody, V., \& } \\
\text { Jones S. }\end{array}$ & 23 & $\begin{array}{l}\text { The case of EAI in facilitating e- } \\
\text { government services in a Welsh } \\
\text { authority }\end{array}$ & $\begin{array}{l}\text { International } \\
\text { Journal of } \\
\text { Information } \\
\text { Management }\end{array}$ & 2009 \\
\hline $\begin{array}{l}\text { van Velsen, L., van der } \\
\text { Geest, T., ter Hedde, } \\
\text { M., \& Derks, W. }\end{array}$ & 22 & $\begin{array}{l}\text { Requirements engineering for e- } \\
\text { government services: A citizen- } \\
\text { centric approach and case study }\end{array}$ & $\begin{array}{l}\text { Government } \\
\text { Information } \\
\text { Quarterly }\end{array}$ & 2009 \\
\hline $\begin{array}{l}\text { Panopoulou, E., } \\
\text { Tambouris, E., \& } \\
\text { Tarabanis, K. }\end{array}$ & 21 & $\begin{array}{l}\text { A framework for evaluating web sites } \\
\text { of public authorities }\end{array}$ & $\begin{array}{l}\text { Aslib } \\
\text { Proceedings: } \\
\text { New Information } \\
\text { Perspectives }\end{array}$ & 2008 \\
\hline Total Geral & 681 & & & \\
\hline
\end{tabular}

Fonte: Dados da Pesquisa

No artigo mais citado, Tabela 5, analisou-se a aceitação dos usuários à implantação de ferramentas de e-serviços; no segundo mais citado, verificou-se a percepção de utilidades, riscos e confiança na adoção dessa 
ferramenta. Nestes estudos, foram analisados casos específicos de implantação em países, como Holanda, Singapura, Malásia, Reino Unido. Dois trabalhos apresentaram marco teórico de implantação, planejamento e avaliação do e-serviços.

Na Tabela 6, apresentam-se as revistas por número de publicações, sendo Electronic Government, Government Information Quarterly e International Journal of Electronic Government Research as principais revistas que publicaram essa temática.

Tabela 6: Número de publicações por revista

\begin{tabular}{l|c}
\hline \multicolumn{1}{c}{ Publicação } & Quantidade Total \\
\hline Electronic Government & 10 \\
\hline Government Information Quarterly & 9 \\
\hline International Journal of Electronic Government Research & 9 \\
\hline International Journal of Information Management & 3 \\
\hline Behaviour and Information Technology & 3 \\
\hline International Journal of Electronic Governance & 3 \\
\hline Transforming Government People Process and Policy & 2 \\
\hline IFIP Advances in Information and Communication Technology & 2 \\
\hline Decision Support Systems & 2 \\
\hline International Journal of Applied Systemic Studies & 2 \\
\hline Information and Management & 2 \\
\hline Journal of Cases on Information Technology & 2 \\
\hline Journal of Organizational and End User Computing & 2 \\
\hline Telecommunications Policy & 2 \\
\hline Transylvanian Review of Administrative Sciences & 1 \\
\hline European Journal of Economics Finance and Administrative Sciences & 1 \\
\hline European Journal of Information Systems & 1 \\
\hline European Journal of Social Sciences & 1 \\
\hline IEEE Technology and Society Magazine & 24 \\
\hline Outros & $\mathbf{8 6}$ \\
\hline Total Geral & \\
\hline
\end{tabular}

Fonte: Dados da Pesquisa

Na Tabela 7, apresenta-se o número de publicação por eventos, sendo o European Conference on e-Government - ECEG, o principal momento de discussão desta temática, com 64 trabalhos apresentados. 
Tabela 7: Número de publicações por eventos

\begin{tabular}{|c|c|}
\hline Evento & Quantidade \\
\hline European Conference on e-Government - ECEG & 64 \\
\hline IFIP Advances in Information and Communication Technology & 11 \\
\hline International Conference on Electronic Business - ICEB & 9 \\
\hline Americas Conference on Information Systems - AMCIS & 8 \\
\hline Americas Conference on Information Systems - AMCIS & 8 \\
\hline Lecture Notes in Business Information Processing & 7 \\
\hline 2011 International Conference on E-Business and E-Government - ICEE & 4 \\
\hline International Conference on Management and Service Science - MASS 2009 & 4 \\
\hline Evento & Quantidade \\
\hline $\begin{array}{l}\text { Innovation and Knowledge Management: A Global Competitive Advantage - } \\
\text { Proceedings of the 16th International Business Information Management } \\
\text { Association Conference - IBIMA } 2011\end{array}$ & 3 \\
\hline International Conference on E-Business and E-Government - ICEE 2010 & 3 \\
\hline 2010 IST-Africa & 3 \\
\hline $\begin{array}{l}\text { Proceedings of the European, Mediterranean and Middle Eastern Conference } \\
\text { on Information Systems: Global Information Systems Challenges in } \\
\text { Management - EMCIS } 2010\end{array}$ & 3 \\
\hline $\begin{array}{l}2009 \text { International Conference on Information Management, Innovation } \\
\text { Management and Industrial Engineering - ICIII } 2009\end{array}$ & 3 \\
\hline $\begin{array}{l}2008 \text { 3rd International Conference on Information and Communication } \\
\text { Technologies: From Theory to Applications - ICTTA }\end{array}$ & 3 \\
\hline $\begin{array}{l}2014 \text { 1st International Conference on e-Democracy and e-Government - } \\
\text { ICEDEG } 2014\end{array}$ & 2 \\
\hline Outros & 78 \\
\hline Total Geral & 213 \\
\hline
\end{tabular}

Fonte: Dados da pesquisa

Com este estudo bibliométrico, pode-se perceber a evolução nesses 15 anos dos trabalhos de governo eletrônico na perspectiva de e-serviços: houve crescente apresentação em eventos de 2005 a 2011; e de 2009 a 2013, publicações definitivas em revistas. Apresentaram-se trabalhos com mais de 100 citações; Reino Unido, Estados Unidos da América e China tiveram autores com mais produção, e o Brasil esteve presente entre os 19 países com mais de três publicações.

\section{CONCLUSÃO}

Neste trabalho, o objetivo foi analisar a produção e o perfil das publicações do tema ferramentas de e-serviços referente a governo eletrônico na base de dados Scopus, no período de 2001 a 2015. Para tal análise, foi 
realizado um levantamento bibliométrico, fundamentado em dados descritivos e quantitativos, no qual foram analisados 299 artigos publicados na base Scopus.

No tema governo eletrônico, o tópico e-serviços é o ponto que tem ligação direta com a atividade dos contribuintes, principalmente as empresas, com ferramentas para controle, apuração e fiscalização contábil e tributária. Com incentivo da Organização das Nações Unidas, países de todos os continentes, de todos os estágios de desenvolvimento, estão implantando e organizando essa relação governo-negócios.

Concluiu-se pelos resultados encontrados que o tema teve aumento nas publicações em eventos até 2010, com 45 trabalhos, e em revista até 2013, com 16 trabalhos. Observou-se também que nos primeiros anos, ou seja, de 2001 a 2004, as publicações referentes ao tema tiveram um pequeno número e concentraram-se em eventos, e apenas no ano de 2002 não houve nenhuma publicação.

Conforme levantamento efetuado, constatou-se que Ciências da Computação e Negócios, Gestão e Contabilidade são as principais áreas dessas publicações. Quanto à distribuição geográfica dos autores, destacaram-se Reino Unido, Estados Unidos da América e China. Entre os trabalhos mais citados, destacam-se estudos que verificaram a implantação e a adoção das ferramentas, em países.

Como limitação do estudo, destaca-se a utilização de uma só base de dados. Sugere-se, para futuros trabalhos em relação ao tema pesquisado, incluir outras bases de dados, aumentando assim o número de artigos a serem pesquisados, a análise das metodologias e as teorias utilizadas nos trabalhos. 


\section{REFERÊNCIAS}

Agune, R., \& Carlos, J. (2005). Governo eletrônico e novos processos de trabalho.In E. Levy, \& P. Drago, Gestão pública no Brasil contemporâneo. São Paulo: Fundap.

Brasil. (2009). Ajuste SINIEF 02/2009. Dispõe sobre a Escrituração Fiscal Digital - EFD. Recuperado em 21 de outubro, 2014, de http://www1.fazenda.gov.br/confaz/confaz/Ajustes/2009/AJ 002 09.ht $\underline{\mathrm{m}}$

Britto (2008).

Chahin, A., Cunha, M. A., Knight, P. T., \& Pinto, S. L. (2004). E-gov.br-a próxima revolução brasileira - eficiência, qualidade e democracia: o governo eletrônico no Brasil e no mundo. São Paulo: Pearson Prentice Hall.

Chen, Y. N., Chen, H. M., Huang, W., \& Ching, R. K. (2006). E-government strategies in developed and developing countries: an implementation framework and case study. Journal of Global Information Management JGIM, 14(1), 23-46.

Cunha, M. A. V. C. (2005). Meios eletrônicos e transparência: a interação do vereador brasileiro com o cidadão e o poder executivo. Anais do Congresso Internacional del CLAD sobre la Reforma del Estado y de La Administración Pública, 10, Santiago, Chile.

Diniz, E. H., Barbosa, A. F., Junqueira, A. R. B., \& Prado, O. (2009). O governo eletrônico no Brasil: perspectiva histórica a partir de um modelo estruturado de análise. Revista de Administração Pública, 43(1), 23-48.

Geron, C. M. S., Finatelli, J. R., Faria, A. C. de, \& Carmo Romeiro, M. do. (2011). SPED-Sistema Público de Escrituração Digital: percepção dos contribuintes em relação os impactos de sua adoção. Revista de Educação e Pesquisa em Contabilidade - REPeC, 5(2), 44-67.

Helbig, N. C., Gil-García, J. R., \& Ferro, E. (2005). Understanding the complexity in electronic government: implications from the digital divide literature. Proceedings of the Americas Conference on Information Systems - AMCIS, 11, Omaha, NE, USA.

Knight, P. T., Fernandes, C. C. C., \& Cunha, M. A. (Orgs.). (2007). Edesenvolvimento no Brasil e no mundo: subsídios e programas e-Brasil. São Paulo: Yends. 
Levy, E., \& Drago, P. A. (Eds.). (2005). Gestão pública no Brasil contemporâneo. São Paulo: Fundap.

Matos, D. S., Costa, G. B. da, Pereira, S. A., \& Locks, R. (2008).

Governança eletrônica na administração pública: estudo de caso sobre a nota fiscal eletrônica-NF-E. Anais do Congresso Brasileiro de Contabilidade, 18, Gramado, RS, Brasil.

Mello, G. R. (2009). Estudo das práticas de governança eletrônica: instrumento de controladoria para a tomada de decisões na gestão dos estados brasileiros. Tese de Doutorado, Faculdade de Economia, Administração e Contabilidade, Universidade de São Paulo, SP, Brasil.

Menezes, J. P. C. B. (2012). Governo eletrônico: introdução do SPED em Portugal baseado no modelo brasileiro. Revista Economia \& Gestão, 12(29), 4-16.

Mezzaroba, O., \& Galindo, F. (2010). Democracia eletrônica. Zaragoza: Prensas Universitarias de Zaragoza. 284 p.

Pessi, P. (2007). Serviços públicos por meios eletrônicos - uma visão orientada ao cidadão. In P. T. Knight, C. C. C. Fernandes, \& M. A. Cunha (Orgs.)., E-desenvolvimento no Brasil e no mundo: subsídios e programa E-Brasil (pp. 538-558). São Caetano do Sul: Yendis.

Ruelas, A. L., \& Arámburo, P. P. (2006, julio). El gobierno electrónico: su estudio y perspectivas de desarrollo. UNIrevista, 1 (3.

Rezende, D. A., Frey, K., \& Betini, R. C. (2003). Governança e democracia eletrônica na gestão urbana.Anais do Seminário Internacional em Gestão Urbana, 1, Curitiba, PR, Brasil/ Compiègne, France.

Santhanamery, T., \& Ramayah, T. (2012). Tax payers continued use of an e-filing system: a proposed model. Technics Technologies Education Management, 7(1), 249-258.

Sepúlveda, M. A. T., Vásquez, A. V., \& Gutiérres, P. G. (2006). Gobierno electronico en Chile 2000-2005. Chile: Maval.

Silva, H. P., \& Lima, J. B. (2007). Governo eletrônico e informação utilitária: uma relação necessária para uma efetiva inclusão digital.

United Nations Educational, Scientific And Cultural Organization - Unesco. (2005). Defining E-governance. Local: Unesco.

Vanti, N. A. P. (2002). Da bibliometria à webometria: uma exploração conceitual dos mecanismos utilizados para medir o registro da 
informação e a difusão do conhecimento. Ciência da Informação, 31(2), 152-162.

Vanz, S. A. D., \& Stumpf, I. R. C. (2010). Procedures and tools applied to bibliometric studies. Informação \& Sociedade - Estudos, 20(2), 67-75.

Zweers, K., \& Planqué, K. (2001). Electronic government. From a organizational based perspective towards a client oriented approach. In J. E. J. Prins (Ed.), Designing E-government (p. 92).The Hauge, The Netherlands: Kluwer Law International. 